\title{
Schistosoma haematobium- Schistosoma mansoni Hybrid Parasite in Migrant Boy, France, 2017
}

\author{
Yohann Le Govic, Julien Kincaid-Smith, \\ Jean-François Allienne, Olivier Rey, \\ Ludovic de Gentile, Jérôme Boissier
}

Author affiliations: Université Bretagne Loire, Angers, France (Y. Le Govic); Centre Hospitalier Universitaire d'Angers, Angers (Y. Le Govic, L. de Gentile); Université de Montpellier, Perpignan, France (J. Kincaid-Smith, J.-F. Allienne, O. Rey, J. Boissier)

DOI: https://doi.org/10.3201/eid2502.172028

Schistosomiasis is frequently detected in persons entering Europe. In 2017, we detected a Schistosoma mansoniSchistosoma haematobium hybrid parasite infection in a migrant boy from Côte d'Ivoire entering France. Because such parasites might be established in Europe, as illustrated by an outbreak on Corsica Island, vectors of these parasites should be investigated.

$\mathrm{I}$ n 2017, a 14-year-old boy from Côte d'Ivoire who had crossed the Sahara Desert through Niger and reached Europe by sea from Libya was referred to the Consultation Board at the Parasitology Unit of Angers University Hospital (Angers, France) for painless gross hematuria. Aside from hematuria, his physical examination was unremarkable. Blood tests revealed microcytic anemia (hemoglobin $103 \mathrm{~g} / \mathrm{L}$, mean corpuscular volume $57.1 \mathrm{fL}$, mean corpuscular hemoglobin concentration $285 \mathrm{~g} / \mathrm{L}$ ) and a low serum ferritin level $(8 \mu \mathrm{g} / \mathrm{L})$. Leukocyte and eosinophil counts and biochemical markers of liver and kidney function were within reference limits. Serologic test results were negative for antibodies to HIV, hepatitis B and C virus, and Treponema pallidum. Serodiagnostic test results were negative for cystic and alveolar echinococcoses and strongyloidiasis, and schistosomiasis screening test results were inconclusive: positive by Schistosoma mansoni-specific ELISA (optical density 1.02, threshold 0.53; Bordier Affinity Products, http:/www.bordier.ch/) and negative by S. mansoni indirect hemagglutination test (titer 1:40, threshold 1:160; Bilharziosis Fumouze; https://www.biosynex.com/).

To clarify diagnosis, we performed the SCHISTO II Western Blot IgG (LDBio Diagnostics, http://www. ldbiodiagnostics.com/), which showed 5 unequivocal bands, including a large band at 22-24 kDa and 30-34 kDa, results indicative of schistosomiasis. We corroborated our results by microscopic examination of patient feces and urine. We detected lateral-spined eggs (typical of $S$. mansoni parasites) in a fecal specimen processed by using the Kato-Katz method and, surprisingly, lateral-spined eggs and terminal-spined eggs (typical of Schistosoma haematobium parasites) in a 24-hour urine specimen filtered through a 12-micron membrane (Figure, panel A). The patient received a single $40 \mathrm{mg} / \mathrm{kg}$ dose of praziquantel. The patient experienced no more episodes of hematuria for the following 6 months; however, we could not assess his parasitological responses.

We genotyped both terminal- and lateral-spined eggs individually using a previously described protocol (1). All terminal-spined eggs were characterized by mitochondrial cox 1 genes (GenBank accession nos. MG562514-5) and the nuclear internal transcribed spacer (ITS) (GenBank accession no. MG554667) specific to S. haematobium schistosomes. All chromatograms of ITS genes from lateral-spined eggs showed a double profile: 1 identical to $S$. mansoni schistosomes (GenBank accession no. MG554659) and 1 identical to $S$. haematobium (GenBank accession no. MG554667). Moreover, the cox 1 haplotypes of these eggs were either specific to $S$. haematobium (GenBank accession no. MG562514) or S. mansoni (GenBank accession nos. MG562512-3) parasites. The phylogenetic tree of $S$. mansoni cox 1 sequences indicates that the parasite responsible for this infection originated from Far West Africa; the hybrid parasite's haplotypes clustered with those of schistosomes from Niger, Senegal, and Mali (Figure, panel B).

Schistosomiasis represents a serious disease burden worldwide and is ranked the 12th most common travel-associated infection in Europe (2). The migration crisis has led to a large flow of persons (notably children) from West Africa. Unaccompanied foreign minors are protected under the 1989 United Nations Convention on the Rights of the Child (https:// www.ohchr.org/en/professionalinterest/pages/crc.aspx). Moreover, because of the high prevalence of schistosomiasis in their countries of origin, these children receive care for this disease upon their arrival in Maine-et-Loire Department (Loire Valley, France). This preventive strategy enabled us to diagnose schistosomiasis in $\approx 25 \%$ of travelers and migrants (Y. Le Govic, unpub. data), a result in accordance with a similar screening program in Italy (3). This strategy also enabled us to detect the case of mixed Schistosoma parasite infection with ectopic egg elimination described in this report.

Ectopic egg elimination (i.e., S. haematobium schistosome eggs in feces and S. mansoni eggs in urine) frequently occurs in endemic areas; in a study in northern Senegal, $53 \%$ of patients infected with Schistosoma parasites had simultaneous infections with $S$. mansoni and S. haematobium parasites, of which $15 \%$ displayed ectopic egg elimination (4). This phenomenon can occur because of parasite hybridization. Hybrids resulting from $S$. mansoni and S. haematobium schistosome cross-breeding have been documented in northern Senegal (5). The fact that the patient we describe 

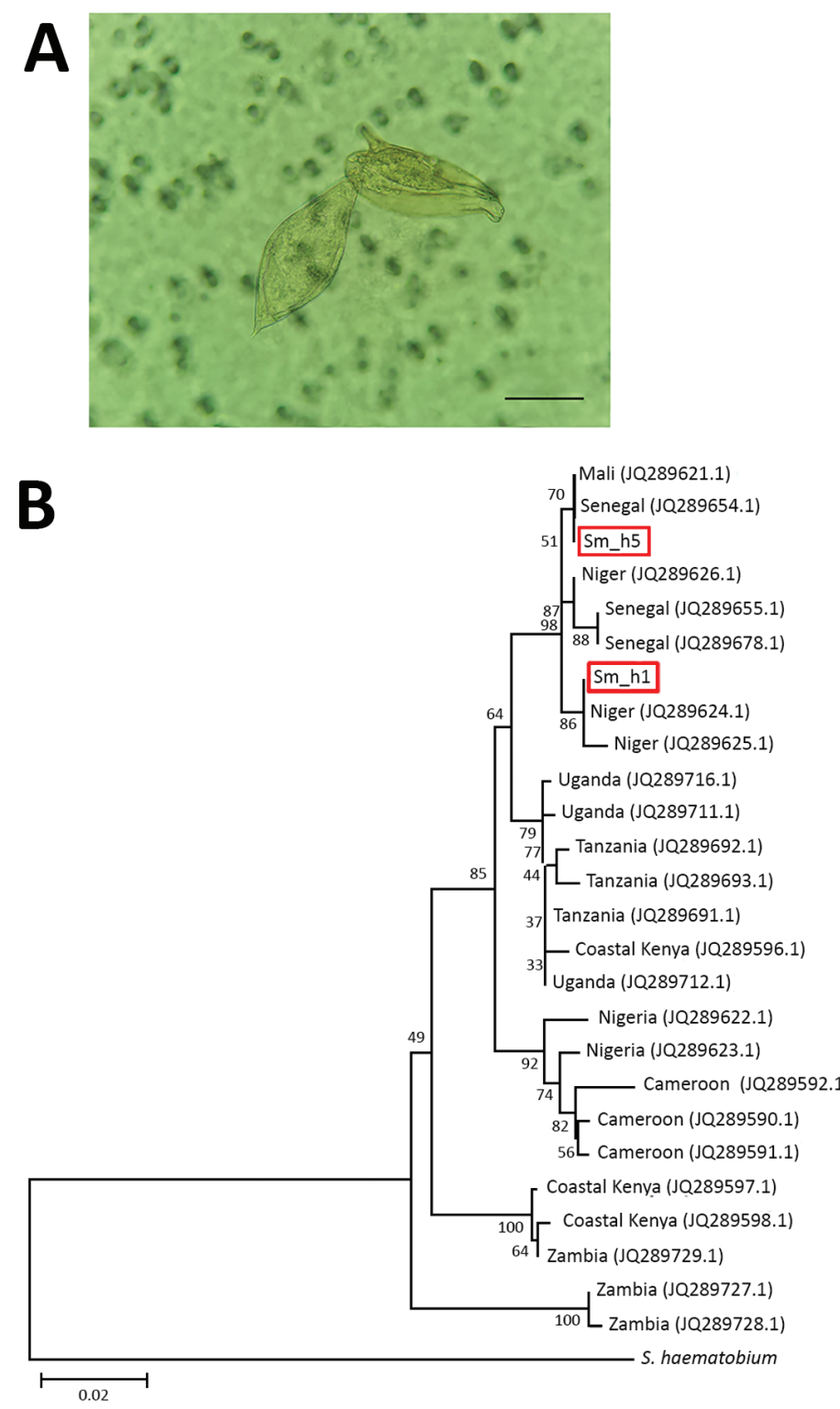

Figure. Characterization of Schistosoma parasites detected in 14-year-old migrant boy from Côte d'Ivoire in France, 2017.

A) Co-detection of terminalspined schistosome eggs (typical of Schistosoma haematobium parasites) and lateral-spined schistosome eggs (typical of Schistosoma mansoni parasites) in urine sample from migrant boy. Sample was microscopically examined after filtration. Original magnification $\times 400$. Scale bar represents $50 \mu \mathrm{m}$. B) Phylogenetic analysis of $S$. mansoni cox 1 gene haplotypes present in migrant boy (boxes). Tree was constructed by using the neighbor-joining method,

Far West Africa the Hasegawa-Kishino-Yano plus gamma distribution model, and 1,000 bootstrap replicates. GenBank accession numbers of haplotypes sampled are provided. Scale bar indicates nucleotide substitutions per residue.
East Africa

Central Africa

Central Africa

East Africa never traveled through Senegal strongly suggests that such hybrids are more widespread than previously observed.

Hybrid schistosomes are particularly worrisome. The outbreak in Corsica Island involved infections with $S$. haematobium-Schistosoma bovis hybrid parasites $(1,6)$, which might be more difficult to diagnose (7). Experimental studies have revealed that interspecific hybridization might enhance infectivity, virulence, and longevity and accelerate cercarial maturation. Also, hybrids can have wider host spectrums, potentially expanding their epidemiologic consequences (8).

The risk for emergence of $S$. mansoni schistosome infection might exist in Europe; the Biomphalaria snail vector (namely B. tenagophila) has been detected for several years in Romania (9). Given the nonnegligible prevalence of mansonic schistosomiasis in travelers and migrants entering Europe $(3,10)$ and global warming, the probability of encounters between $S$. mansoni miracidia and their snail hosts might have increased. Moreover, whether $S$. mansoni-S. haematobium hybrid parasites are capable of infecting the Bulinus snail vector of the S. haematobium schistosome, which is widely distributed throughout Europe (France, Spain, Italy, Greece, Portugal) (11), remains unknown and deserves further investigation.

\section{About the Author}

Dr. Le Govic is a clinical biologist and researcher in Angers University Hospital Center, University of Angers, Angers, France. His primary research interests include emerging fungal and parasitic infections, with a special emphasis on epidemiology, pathophysiology, and diagnosis. 


\section{References}

1. Boissier J, Grech-Angelini S, Webster BL, Allienne JF, Huyse T, Mas-Coma S, et al. Outbreak of urogenital schistosomiasis in Corsica (France): an epidemiological case study. Lancet Infect Dis. 2016;16:971-9. http://dx.doi.org/10.1016/ S1473-3099(16)00175-4

2. Schlagenhauf P, Weld L, Goorhuis A, Gautret P, Weber R, von Sonnenburg F, et al.; EuroTravNet. Travel-associated infection presenting in Europe (2008-12): an analysis of EuroTravNet longitudinal, surveillance data, and evaluation of the effect of the pre-travel consultation. Lancet Infect Dis. 2015; 15:55-64. http://dx.doi.org/10.1016/S1473-3099(14)71000-X

3. Beltrame A, Buonfrate D, Gobbi F, Angheben A, Marchese V, Monteiro GB, et al. The hidden epidemic of schistosomiasis in recent African immigrants and asylum seekers to Italy. Eur J Epidemiol. 2017;32:733-5. http://dx.doi.org/10.1007/ s10654-017-0259-6

4. Meurs L, Mbow M, Vereecken K, Menten J, Mboup S, Polman K. Epidemiology of mixed Schistosoma mansoni and Schistosoma haematobium infections in northern Senegal. Int J Parasitol. 2012;42:305-11. http://dx.doi.org/10.1016/j.ijpara.2012.02.002

5. Huyse T, Van den Broeck F, Hellemans B, Volckaert FA, Polman K. Hybridisation between the two major African schistosome species of humans. Int J Parasitol. 2013;43:687-9. http://dx.doi.org/10.1016/j.ijpara.2013.04.001

6. Moné H, Holtfreter MC, Allienne JF, Mintsa-Nguéma R, Ibikounlé M, Boissier J, et al. Introgressive hybridizations of Schistosoma haematobium by Schistosoma bovis at the origin of the first case report of schistosomiasis in Corsica (France, Europe). Parasitol Res. 2015;114:4127-33. http://dx.doi.org/10.1007/ s00436-015-4643-4

7. Moné H, Holtfreter MC, Mouahid G, Richter J. Difficulties in schistosomiasis assessment, Corsica, France. Emerg Infect Dis. 2016;22:762-3. http://dx.doi.org/10.3201/eid2204.160110

8. Webster BL, Southgate VR. Compatibility of Schistosoma haematobium, S. intercalatum and their hybrids with Bulinus truncatus and B. forskalii. Parasitology. 2003;127:231-42. http://dx.doi.org/10.1017/S0031182003003597

9. Majoros G, Fehér Z, Deli T, Földvári G. Establishment of Biomphalaria tenagophila snails in Europe. Emerg Infect Dis. 2008;14:1812-4. http://dx.doi.org/10.3201/eid1411.080479

10. Lingscheid T, Kurth F, Clerinx J, Marocco S, Trevino B, Schunk M, et al.; TropNet Schistosomiasis Investigator Group. Schistosomiasis in European travelers and migrants: analysis of 14 years TropNet surveillance data. Am J Trop Med Hyg. 2017;97:567-74. http://dx.doi.org/10.4269/ajtmh.17-0034

11. Welter-Schultes F. European non-marine molluscs, a guide for species identification. Gottingen, Germany: Planet Poster Editions; 2012.

Address for correspondence: Jérôme Boissier, CNRS, UMR 5244, 52 Avenue Paul Alduy, Perpignan 66000, France; email: boissier@univ-perp.fr

\section{West Nile Virus Infection in Travelers Returning to United Kingdom from South Africa}

\author{
Vivak Parkash, Kate Woods, \\ Liana Kafetzopoulou, Jane Osborne, \\ Emma Aarons, Katharine Cartwright
}

Author affiliations: Royal Hallamshire Hospital, Sheffield, UK (V. Parkash, K. Cartwright); Public Health England, Porton Down, UK (K. Woods, L. Kafetzopoulou, J. Osborne, E. Aarons)

DOI: https://doi.org/10.3201/eid2502.172101

West Nile virus (WNV) is an arthropod-transmitted flavivirus that causes West Nile fever and may infrequently cause neuroinvasive disease in humans. We present 2 cases of confirmed WNV infection, 1 of severe encephalitis and 1 of mild febrile illness, in a couple returning to the United Kingdom from South Africa.

$\mathrm{W}$ est Nile virus (WNV) is a mosquitoborne flavivirus maintained in an enzootic cycle between culicine mosquitoes and birds. Approximately $80 \%$ of human infections are subclinical, although symptomatic cases can vary from influenza-like symptoms to neurotropic manifestations (1). West Nile neuroinvasive disease, which manifests as encephalitis, meningitis, or acute flaccid paralysis, occurs in $<1 \%$ of all cases (2).

To date, human disease caused by WNV has been attributed to WNV phylogenetic lineages 1 and 2 (3). Lineage 2 consists of viruses found in South Africa, Madagascar, and, more recently, in Europe and Russia (4). Lineage 2 was previously described as less pathogenic than lineage 1 , but it has subsequently been demonstrated that lineage 2 can also lead to neuroinvasive disease (5). We describe a couple from the United Kingdom with confirmed WNV infection who were admitted to a hospital in February 2017 on return from a holiday to South Africa, where they most likely acquired WNV in the Kruger National Park region.

A previously healthy 76-year-old woman was admitted with acute confusion to Doncaster Royal Infirmary, Doncaster, UK. She and her husband had returned that day following a 3-week holiday visiting family in South Africa (Appendix Figure, http://wwwnc.cdc.gov/EID/article/25/2/172101-App1.pdf). The patient and her husband had stayed in Johannesburg, except for a 5-day safari in Kruger National Park 5 days into their trip. They had both sustained several mosquito and tick bites. No malaria prophylaxis had been taken, and no pretravel advice had been sought.

The patient boarded an airplane independently in Johannesburg but her condition deteriorated in flight; on arrival, 